Revue européenne des sciences sociales

\title{
Le temps des manifestations
}

\section{Nonna Mayer}

\section{OpenEdition}

\section{Journals}

Édition électronique

URL : http://journals.openedition.org/ress/410

DOI : $10.4000 /$ ress. 410

ISSN : 1663-4446

\section{Éditeur}

Librairie Droz

\section{Édition imprimée}

Date de publication : 1 mars 2004

Pagination : 219-224

ISBN : 2-600-00941-8

ISSN : 0048-8046

Référence électronique

Nonna Mayer, "Le temps des manifestations », Revue européenne des sciences sociales [En ligne], XLII-129 | 2004, mis en ligne le 05 novembre 2009, consulté le 22 avril 2019. URL : http:// journals.openedition.org/ress/410; DOI : 10.4000/ress.410 


\section{Nonna MAYER}

\section{LE TEMPS DES MANIFESTATIONS}

Comme toute activité sociale, la contestation a son temps propre. Elle a ses jours. Les manifestations de rue sont plus fréquentes en semaine, avec un pic le jeudi, même si celles du dimanche attirent plus de monde ${ }^{1}$. Elle a ses saisons. «L'hiver est le temps du repli sur soi, alors que le printemps est propice aux explosions revendicatives avec ses rythmes traditionnels de contestation», note Philippe Besnard dans son enquête sur les cadences et les rythmes de la vie collective des Français. Révoltes paysannes de l'Ancien Régime, grèves ouvrières et révoltes urbaines du XIX ${ }^{\mathrm{e}}$, toutes ces actions connaissent le même pic printanier ${ }^{2}$. Si les congés payés sont venus bouleverser le rythme des mouvement sociaux, aujourd'hui encore grèves et manifestations de rue se multiplient en mai-juin, désormais encadrées toutefois par deux autres saisons contestataires, l'une en mars, l'autre en septembre-octobre, la rentrée sociale succédant à la grande pause estivale $^{3}$. Il y a enfin des périodes plus effervescentes que d'autres, porteuses de cycles de protestation comme les années trente, mai 68, ou l'automne 95. Mais globalement la contestation tend à se développer dans les démocraties occidentales, qu'on en juge par le nombre de manifestations recensées ${ }^{4}$, la proportion de personnes déclarant avoir déjà participé à des manifestations de rue ${ }^{5}$ ou simplement prêtes à le faire, pour défendre leurs opinions ou leurs revendications. C'est cette légitimité croissante de la manifestation de rue, définie au sens large comme occupation collective de l'espace public à des fins d'expression politique, qui retient ici notre attention.

Olivier Fillieule, Stratégies de la rue. Les manifestations en France, Paris, Presses de Sciences Po, 1997, p. 82 et Danielle Tartakowsky, Les manifestations de rue en France. 1918-1968, Paris, Publications de la Sorbonne, 1997, p. 633.

$2 \quad$ Philippe Besnard, Mours et humeurs des Français au fil des saisons, Paris, Balland, 1989, p. 124. Voir notamment le graphique établi à partir des données fournies par Mark Traugott sur l'édification des barricades en France entre 1788 et 1871, p. 110.

3 Besnard, op. cit., p.125; Fillieule, op. cit., p. 75-77.

$4 \quad$ Le dépouillement des archives policières pour les villes de Nantes et Marseille durant les années 80 réalisé par Olivier Fillieule montre une augmentation régulière du nombre de manifestations. Dans le même temps toutefois le nombre moyen de manifestants a baissé, les manifestations perdent de leur ampleur au profit de nombreuses micro-mobilisations (op. cit. p. 71-75).

5 Sur la hausse du nombre de personnes déclarant avoir déjà manifesté voir les enquêtes comparatives «Valeurs», présentées dans un numéro spécial de la revue Futuribles, juillet-août 2002, $\mathrm{n}^{\circ} 277$, et l'enquête européenne «Beliefs in government», notamment l'analyse de Richard Topf, «Beyond electoral participation», in Hans Dieter Klingemann et Dieter Fuchs (dir.), Citizens and the State, Oxford, Oxford University Press, 1995, p. 27-51. 


\section{LE POTENTIEL PROTESTATAIRE}

Dans leur travail pionnier sur la transformation des formes de participation politique dans les démocraties occidentales, révélée par les «nouveaux mouvements sociaux » des années soixante, Barnes et Kaase ${ }^{6}$ notaient la routinisation des modes d'action collective initiés par le mouvement ouvrier: tels que grève, manifestation de rue, occupation de locaux, etc., et leur diffusion au sein des classes moyennes et supérieures. Ils liaient l'essor d'un «potentiel protestataire», mesuré par la proportion de la population approuvant le recours à de tels moyens, aux transformations de la société post industrielle. La hausse du niveau d'étude, la montée des valeurs «post matérialistes » centrées sur l'épanouissement individuel et le refus de l'autorité, rendraient les citoyens plus autonomes et plus critiques. Elles les inciteraient à interpeller directement les gouvernants, sans passer par la médiation des représentants élus, et favoriseraient la multiplication des demandes sectorielles.

Le cas de la France est à cet égard exemplaire. Depuis la fin des années 80 les enquêtes du Cevipof, menées auprès d'échantillons nationaux représentatifs de la population électorale, posent régulièrement une batterie de questions sur les moyens d'action envisagés pour défendre ses opinions ou ses revendications (tableau 1$)^{7}$. Les réponses sont corrélées et permettent de construire une échelle d'attitude, mesurant l'intensité du «potentiel protestataire» selon le nombre et la nature des moyens d'actions envisagés, allant des moins aux plus radicaux ${ }^{8}$. Si une très large majorité des personnes interrogées approuve le principe de la grève ou de la manifestation, moins de la moitié est d'accord pour occuper des locaux ou refuser de payer ses impôts et seule une infime minorité accepte de peindre des slogans sur les murs ou de provoquer des dégâts matériels. A l'exception des deux derniers modes d'action, tous les autres ont vu leur légitimité considérablement progresser d'une enquête à l'autre. Aujourd'hui moins d'un électeur sur dix n'envisage aucun des six moyens proposés. Et la manifestation de rue bat tous les records de popularité. En 1988, un Français sur deux était prêt à y recourir. En 1995 la proportion passait à deux sur trois, et en 2002 elle dépassait les trois quarts, soit une progression record de 27 points, qui met aujourd'hui la manifestation à peu près au même rang que la grève dans la hiérarchie des moyens d'action envisagés (tableau 1).

\footnotetext{
Samuel H. Barnes, Max Kaase (dir.), Political Action. Mass Participation in Five Western Democracies, London, Sage, 1979.

7 Enquêtes administrées par la Sofres, en face à face, échantillons construits sur quotas. Ce sont des enquêtes post électorales pour 1988 et 1995 et pré-electorale pour 2002: vague 1 (8-20 avril) du Panel électoral français 2002, enquête en trois vagues associant le Cevipof (Paris), le Cidsp (Grenoble), et le Cecop (Paris).

8 Une échelle hiérarchique (h de Loevinger). Voir la note méthodologique de Guy Michelat sur «Les échelles d'attitudes et de comportements», in Cevipof, L'électeur français en questions, Paris, Presses de Sciences Po, 1990, p. 229-236. En fait le refus de payer ses impôts relève d'une autre logique contestataire et ne rentre pas dans l'échelle. On le fait figurer pour mémoire sur le tableau 1 .
} 
Tableau 1. Les modes d'action protestataires (\%)

«Voici un certain nombre de moyens que les gens utilisent parfois pour faire connaître leurs opinions ou leurs revendications. Pouvez-vous me dire pour chacun d'eux si vous l'approuveriez ou pas du moins dans certaines circonstances?»

\begin{tabular}{|l|c|c|c|c|}
\hline$\%$ & 1988 & 1995 & 2002 & Ecart \\
\hline Provoquer des dégâts matériels & 1 & 2 & 2 & +1 \\
\hline Peindre des slogans sur les murs & 6 & 6 & 5 & 0 \\
\hline Refuser de payer les impôts & 23 & 37 & 32 & +8 \\
\hline Occuper un bâtiment administratif & 28 & 42 & 43 & +15 \\
\hline Participer à des manifestations de rues & 49 & 62 & 77 & +27 \\
\hline Faire grève & 66 & 74 & 79 & +13 \\
\hline & $(3847)$ & $(4078)$ & $(4107)$ & \\
\hline
\end{tabular}

Enquêtes CEVIPOF 1988/1995 et Panel électoral français 2002, vague 1. Population métropolitaine inscrite sur les listes électorales.

\section{LA DYNAMIQUE MANIFESTANTE}

Cette dynamique est portée par le renouvellement générationnel, comme le montre une analyse par cohortes, suivant l'évolution des opinions à l'égard de la manifestation selon l'année de naissance, de l'élection présidentielle de 1988 à celle de 2002 (tableau 2). Quelle que soit la période considérée, les jeunes se montrent plus enclins à soutenir la manifestation, activité physique et parfois violente. On note de 40 à 50 points d'écart entre le «potentiel manifestant» avant 25 ans et à partir de 74 ans. Mais quelle que soit l'année de naissance, tout au long de la période considérée, l'acceptation de cette forme d'action a globalement progressé. Chaque nouvelle cohorte qui atteint l'âge de la majorité électorale se montre plus attachée à ce mode d'action que la précédente au même âge. Et en vieillissant les générations précédentes s'y sont converties en proportion croissante. En 1988 la manifestation n'était majoritairement acceptée que chez les générations nées depuis 1943. En 1995 elle le devient chez celles de la guerre et de l'immédiate avant guerre (nées entre 1936 et 1942) et en 2002 chez pratiquement toutes les générations.

Depuis les années 80 , la manifestation a progressivement conquis sa légitimité dans toutes les catégories de la population. C'est aujourd'hui un mode d'action majoritairement approuvé dans toutes les catégories socioprofessionnelles, même chez les indépendants encore très réticents en 1988 (tableau 3). Si les femmes y sont moins favorables que les hommes (respectivement 74 et $80 \%$ d'approbation en 2002), c'est à cause du poids plus marqué des personnes âgées.

Chez les moins de 35 ans, le genre n'entraîne plus de différence. Et si le recours à l'action collective et solidaire pour défendre ses revendications est plus ancré dans la culture de gauche, il s'est considérablement développé chez les 
Tableau 2. Acceptation de la manifestation par cohortes (\%)

\begin{tabular}{|l|c|c|c|c|c|c|}
\hline Année de naissance & Age 1988 & 1988 & 1995 & 2002 & Age 2002 & $\begin{array}{c}\text { Evolution } \\
1988 / 2002\end{array}$ \\
\hline $1978-1984$ & & - & - & 88 & $18-24$ ans & - \\
\hline $1971-1977$ & & - & 83 & 89 & $25-31$ ans & +6 \\
\hline $1964-1970$ & $18-24$ ans & 68 & 74 & 87 & $32-38$ ans & +19 \\
\hline $1957-1963$ & $25-31$ ans & 65 & 73 & 85 & $39-45$ ans & +20 \\
\hline $1950-1956$ & $32-38$ ans & 62 & 71 & 79 & $46-52$ ans & +17 \\
\hline $1943-1949$ & $39-45$ ans & 51 & 61 & 71 & $53-59$ ans & +20 \\
\hline $1936-1942$ & $46-52$ ans & 44 & 51 & 70 & $60-66$ ans & +26 \\
\hline $1929-1935$ & $53-59$ ans & 36 & 47 & 62 & $67-73$ ans & +26 \\
\hline $1922-1928$ & $60-66$ ans & 32 & 40 & 48 & 74 ans + & +16 \\
\hline $1915-1921$ & $67-73$ ans & 30 & 32 & - & & \\
\hline $1908-1914$ & 74 ans + & 23 & - & - & & \\
\hline Total & & 49 & 62 & 77 & & \\
\hline
\end{tabular}

Enquêtes CEVIPOF 1988/1995 et Panel électoral français 2002, vague 1. Population métropolitaine inscrite sur les listes électorales.

électeurs de droite où le taux d'approbation de la manifestation a progressé de plus de 30 points de pourcentage depuis 1988 (tableau 4).

Le «potentiel manifestant» tel que le mesurent ces questions ne se traduira pas nécessairement en actes. La mobilisation effective dépend du travail militant, de la force des réseaux, de l'efficacité de la propagande des organisations qui appellent à manifester' ${ }^{9}$. Et chaque manifestation est un évènement unique, dont la temporalité, ses revendications, le public ne ressemblent à aucun autre ${ }^{10}$. Mais les sondages nous renseignent sur l'adhésion de principe et sur la légitimité de la manifestation comme forme d'action collective. Et ils suggèrent une indéniable familiarisation des jeunes d'aujourd'hui avec la pratique de la manifestation et de la grève (tableau 5). A la veille du scrutin présidentiel du 21 avril 2002, un cinquième des personnes interrogées âgées de 65 ans ou plus disent avoir participé au moins une fois à ce type d'action quand elles avaient entre 15 et 20 ans. La proportion dépasse les deux tiers dans la tranche des 18-24 ans.

\footnotetext{
Sur les deux séquences type des mouvements sociaux, la «mobilisation du consensus » suivie par la «mobilisation de l'action» voir l'article classique de Bert Klandermans et Dirk Oegema, «Potentials, networks, motivations and barriers: steps towards participation in social movements », American Sociological Review, 52, 1987, p. 519-531.

1o Sur la sociologie des évènements manifestants voir Olivier Filleule, op. cit. chapitre 2 et sur la méthodologie du sondage auprès des manifestants, en situation, voir Pierre Favre, Olivier Fillieule, Nonna Mayer, «La fin d'une étrange lacune de la sociologie des mobilisations. L'étude par sondage des manifestants: fondements théoriques et solutions techniques », Revue française de science politique, 47(1), 1997, p. 3-28.
} 
Tableau 3. Acceptation de la manifestation selon la profession et le statut (\%).

\begin{tabular}{|c|c|c|c|c|c|c|c|c|c|c|}
\hline & Agricul. & Patron & Cadre sup & P. interm & Employé & Ouvrier & Chômeur & Etudiant & Privé & Public \\
\hline 1988 & 43 & 31 & 60 & 57 & 48 & 46 & 52 & 81 & 47 & 56 \\
\hline 1995 & 48 & 42 & 75 & 69 & 61 & 59 & 71 & 90 & 62 & 69 \\
\hline 2002 & 65 & 65 & 83 & 83 & 74 & 75 & 80 & 94 & 75 & 83 \\
\hline & +22 & +34 & +23 & +26 & +26 & +29 & +28 & +13 & +28 & +27 \\
\hline
\end{tabular}

Enquêtes CEVIPOF 1988/1995, Panel électoral français 2002, vague 1. Population métropolitaine inscrite sur les listes électorales.

Tableau 4. Acceptation de la manifestation selon la position sur l'échelle gauche droite (\%).

\begin{tabular}{|c|c|c|c|c|c|c|c|c|}
\hline & Gauche 1 & 2 & 3 & 4 & 5 & 6 & 7 Droite & Total \\
\hline 1988 & 75 & 72 & 61 & 42 & 35 & 35 & 37 & 49 \\
\hline 1995 & 74 & 79 & 78 & 60 & 55 & 46 & 40 & 62 \\
\hline 2002 & 91 & 89 & 84 & 73 & 70 & 65 & 67 & 77 \\
\hline & +16 & +17 & +23 & +31 & +35 & +30 & +30 & +28 \\
\hline
\end{tabular}

Enquêtes CEVIPOF 1988/1995 et Panel électoral français 2002, vague 1. Population métropolitaine inscrite sur les listes électorales.

Tableau 5. Pratique de la grève et de la manifestation en fonction de l'âge (\%).

\begin{tabular}{|l|c|c|c|c|c|c|}
\hline \multicolumn{6}{|c|}{ «Entre $\mathbf{1 5}$ et 20 ans, avez-vous participé à une manifestation ou à une grève?» } \\
\hline$\%$ & $18-24$ ans & $25-34$ ans & $35-49$ ans & $50-64$ ans & 65 ans + & Total \\
\hline Jamais & 33 & 39 & 55 & 60 & 79 & 57 \\
\hline Une fois & 25 & 25 & 16 & 14 & 8 & 16 \\
\hline Plusieurs fois & 42 & 36 & 28 & 26 & 12 & 27 \\
\hline SR & 0 & 0 & 1 & 0 & 1 & 0 \\
& $(409)$ & $(735)$ & $(1130)$ & $(916)$ & $(917)$ & $(4107)$ \\
\hline
\end{tabular}

Enquêtes CEVIPOF 1988/1995 et Panel électoral français 2002, vague 1. Population métropolitaine inscrite sur les listes électorales.

Cette hausse du potentiel manifestant s'inscrit dans une tendance de fond, observée dans tous les pays de l'Union européenne. D'après les enquêtes comparatives «Valeurs », entre 1981 et 1999 la proportion d'Européens déclarant avoir déjà suivi une manifestation autorisée est passée de 18 à $27 \%^{11}$. Mais pour

${ }^{11}$ Voir Pierre Bréchon, «Des valeurs politiques entre pérennité et changement », Futuribles, juilletaoût 2002, n 277, p. 106-107. Sur les mobilisations concrètes voir aussi Sidney Tarrow, Doug Imig (dir.), Contentious Europeans, Rowman et Littlefield, 2002. 
diverses raisons, - centralisation de l'Etat, absence d'instances alternatives de régulation et de médiation, fragmentation syndicale et faiblesse de la tradition réformiste, crise de la représentation politique- elle est encore plus marquée en France, qui est dans le peloton de tête avec 38\% de manifestants déclarés en 1999, aux côtés de la Grèce et de la Belgique (respectivement 38 et 39\%). L'ardeur manifestante ne parait donc pas près de s'éteindre.

Il n'est pas inutile de rappeler enfin que dans toutes nos enquêtes ceux qui approuvent et pratiquent la manifestation et la grève sont aussi les plus attachés à la démocratie et à ses institutions, les plus attachés à l'exercice du droit de vote. Loin de s'opposer, ces formes de participation politique se complètent. Elles témoignent de l'élargissement du «répertoire d'action» des citoyens et de leur aspiration à une démocratie plus directe, plus participative, plus forte.

Centre de recherches politiques de Sciences Po (CEVIPOF)-CNRS, Paris mayer@msh-paris.fr 